\title{
Systematic study of the thermal diffusion in associated mixtures
}

\author{
Pavel Polyakov ${ }^{1, *}$ and Simone Wiegand ${ }^{1, \dagger}$ \\ ${ }^{1}$ Forschungszentrum Jülich GmbH, \\ IFF - Weiche Materie, D-52428 Jülich, Germany
}

(Dated: November 5, 2007)

\begin{abstract}
We performed systematic temperature and concentration dependent measurements of the Soret coefficient in different associated binary mixtures of water, deuterated water, dimethyl sulfoxide (DMSO), methanol, ethanol, acetone, methanol, 1-propanol, 2-propanol, propionaldehyde using the so called thermal diffusion forced Rayleigh scattering method. For some of the associating binary mixtures such as ethanol/water, acetone/water and DMSO/water the concentration $x_{w}^{ \pm}$ at which the Soret coefficient changes its sign does not depend on temperature and is equal to the concentration $x_{w}^{\times}$where the Soret coefficient isotherms intersect. While for others such as 1-propanol/water, 2-propanol/water and ethanol/DMSO the sign change concentration is temperature dependent, which is the typical behavior observed for non-associating mixtures. For systems with $x_{w}^{ \pm}=x_{w}^{\times}$we found that $x_{w}^{ \pm}$depends linearly on the ratio of the vaporization enthalpies of the pure components. Probably due to the similarity of methanol and DMSO we do not observe a sign change for this mixture. The obtained results are related to structural changes in the fluid observed by nuclear magnetic resonance, mass specrometric and X-ray experiments in the literature. Furthermore we discuss the influence of hydrophilic and hydrophobic interactions and the solubility on thermal diffusion behavior.

PACS numbers:
\end{abstract}




\section{INTRODUCTION}

Thermal diffusion describes the migration of molecules in a temperature gradient. For a binary mixture in a temperature gradient $\nabla T$, the enrichment of one component $\nabla x$ is characterized by the Soret coefficient $\mathrm{S}_{T}$, as

$$
S_{\mathrm{T}}=-\frac{1}{c(1-c)} \frac{|\nabla c|}{|\nabla T|}
$$

A positive Soret coefficient of the component with the weight fraction $c$ implies that this component moves to the cold region of the fluid.

The main practical applications are separation processes ${ }^{1,2}$ such as thermal field flow fractionation of polymers and colloids or isotope separation, characterization of geochemical processes $^{3,4}$ and combustion ${ }^{5}$. Although the discovery of the effect by Ludwig ${ }^{6}$ dates back 150 years, even qualitative predictions for liquids, which are of practical importance, are often difficult.

Even less than 20 years ago different experimental techniques such as thermo gravitational columns, beam deflection, utilizing diffusion cell and thermal diffusion forced Rayleigh scattering (TDFRS) gave different results for the simple organic mixture of toluene/n-hexane . $^{7-9}$ Later the deviations could be resolved ${ }^{10,11}$ and additionally a benchmark test has been initiated, to measure the Soret coefficient $S_{\mathrm{T}}$ in simple organic mixtures by different experimental techniques $^{12}$. In the recent years it was also demonstrated that the TDFRS method gives reliable results for aqueous systems and compares well with other experimental techniques ${ }^{10,13-15}$. But recent studies on a non-ionic surfactant by the TDFRS technique showed, that the small amount of dye, which is added to convert light into heat energy, can influence the thermal diffusion behavior of the surfactant system ${ }^{16,17}$. Therefore special care needs to be taken for systems with complex phase behavior.

Conceptually, binary mixtures of simple molecules can be divided into three groups: mixture of spherical molecules without specific interactions, mixtures of non-spherical molecules without specific interactions and associated mixtures. In the first group of mixtures the component with the larger mass or higher density moves to the cold side, and this effect becomes stronger if the components are less miscible ${ }^{18-21}$. This empirical observations still hold for some mixtures from the second group ${ }^{18}$ such as benzene/carbon tetrachloride ${ }^{22}$ and cyclohexane/carbon tetrachloride ${ }^{23}$ while for systems benzene/cyclohexane ${ }^{24}$ and benzene/alkanes ${ }^{25}$ 
this approach fails. In order to study the influence of molecular mass more systematically binary mixtures of unpolar solvents with cyclohexane and its isotopes have been investigated ${ }^{26}$. It turned out that the change in $S_{\mathrm{T}}$ after isotopic substitution of cyclohexane, neither depends on concentration nor on the nature of the mixing partner. Only in the case of polar acetone $\Delta \mathrm{S}_{T}$ is approximately $30 \%$ larger but still concentration independent. This investigation has also been extended to a broader temperature range ${ }^{27}$.

Associated mixtures often show a sign change of the Soret coefficient with concentration $^{28-30}$ so that the direction of the thermal diffusion process is predominantly guided by excess properties and not by the properties of the mixing partners like the difference in mass or moment of inertia. Such behavior is expected from the non-ideality of such mixtures due to the hydrogen bond formation. On the other hand it was observed that the sign change concentration correlates with the concentration at which the hydrogen bond network breaks down ${ }^{17}$ and the concentration dependence of the Soret coefficient in aqueous systems seems to be universal ${ }^{31}$. Therefore it might be possible to relate the sign change concentration with properties of the pure components and the structure of the mixture.

The goal of this paper is an investigation of different binary mixtures of polar molecules like water, dimethyl sulfoxide (DMSO), ethanol, acetone, methanol, 1-propanol, 2-propanol, propionaldehyde at different temperatures and concentrations. In order to investigate the mass effect normal water $\mathrm{H}_{2} \mathrm{O}$ was replaced by $\mathrm{D}_{2} \mathrm{O}$. Particular attention has been given to the sign change concentrations. We also looked at the influence of dye (basantol yellow) on the Soret coefficient of ethanol/water mixtures under typical conditions of the TDFRS experiments.

\section{EXPERIMENT}

\section{A. Sample Preparation.}

Methanol (99.8\%), 1-propanol (99.9\%), 2-propanol (99.9\%), DMSO (99.7\%), propionaldehyde $(97 \%)$, deuterated water (99.96 atom \% D) were purchased from Sigma-Aldrich and ethanol (99.5\%) was ordered from Laborchemie Handels-GmbH. We took deionized Milli-Q water. All chemicals were used without further purification. The mixtures were prepared as follows: First a very small amount (roughly $10^{-6}$ wt \%) of the dye basantol yellow ${ }^{16}$, was 
dissolved in the solvents. For each solution, the optical density was adjusted to $2-3 \mathrm{~cm}^{-1}$ at a wavelength of $488 \mathrm{~nm}$. Samples for the TDFRS measurements were prepared just before the measurement to avoid evaporation. The solutions were directly filtered into the sample cells (Spartan, $0.45 \mu \mathrm{m}$ ). The temperature was controlled by a circulating water bath with a temperature stability of $\Delta T= \pm 0.01 \mathrm{~K}$.

\section{B. Refractive index increment measurements.}

Refractive index increments with concentration $(\partial n / \partial c)_{p, T}$ at a constant pressure and temperature were measured using an Abbe refractometer. The temperature derivatives of the refractive index $(\partial n / \partial T)_{p, c}$ at a constant pressure and concentration were determined in a temperature range $T \pm 3{ }^{\circ} \mathrm{C}$ using a Michelson interferometer ${ }^{32}$.

\section{TDFRS experiment and data analysis}

The principle of the TDFRS method is described elsewhere in details ${ }^{33}$. An argon-ion laser $\left(\lambda_{w}=488 \mathrm{~nm}\right)$ is used for writing the temperature grating into the sample. The laser beam is split into two writing beams of equal intensity by a beam splitter. An intensity grating is created in the sample by the interference of two laser beams. A small amount of dye in the sample converts the intensity grating into a temperature grating, which in turn causes a concentration grating by the effect of thermal diffusion. Both gratings contribute to a combined refractive index grating, which is read out by diffraction of a third laser beam $\left(\lambda_{r}=633 \mathrm{~nm}\right)$.

The intensity $\zeta_{\text {het }}(t)$ of the heterodyne signal normalized to the thermal signal is related to the Soret coefficient

$$
\zeta_{\text {het }}(t)=1-A\left(1-e^{-q^{2} D t}\right)
$$

with

$$
A=\left(\frac{\partial n}{\partial T}\right)_{p, c}^{-1}\left(\frac{\partial n}{\partial c}\right)_{p, T} c(1-c) S_{\mathrm{T}}
$$

where $q$ is the grating vector, $D$ is the mutual diffusion coefficient and $A$ is the amplitude of the concentration signal. 
To determine the transport coefficients, Eq. 2 is fitted to the measured heterodyne signal (c.f. Fig. 1a) using two contrast factors $(\partial n / \partial c)_{p, T}$ and $(\partial n / \partial T)_{p, c}$ which are measured separately. The fit residuals are generally less than $1 \%$, even for the mixture with the highest dye content. However a small systematic trend can be observed, which is due to the dye contribution as a third component to the concentration signal. Those systematic deviations vanish, if one accounts for dye contribution by a two mode analysis ${ }^{16,17}$ (c.f. Eq. $3)$.

$$
\begin{aligned}
\zeta_{\text {het }}(t)=1 & -A_{\text {fast }}\left(1-e^{-q^{2} D_{\text {fast }} t}\right) \\
& -A_{\text {slow }}\left(1-e^{-q^{2} D_{\text {slow }} t}\right)
\end{aligned}
$$

Now we want to study in more detail the influence of the added dye and the influence of the laser intensity. Ideally the dye is inert, which means that there is no photo bleaching and no dye contribution to the diffraction signal. In order to study the dye influence, we varied the optical density between $2 \mathrm{~cm}^{-1}-10 \mathrm{~cm}^{-1}$ and investigated the thermal diffusion behavior of ethanol $/ \mathrm{D}_{2} \mathrm{O}$ and ethanol $/ \mathrm{H}_{2} \mathrm{O}$ mixtures with a water mole fraction of 0.697 and 0.954 .

Fig. 1a shows the heterodyne signal for ethanol $/ \mathrm{D}_{2} \mathrm{O}$ for two different optical densities. The dependences of both amplitude and diffusion coefficient versus dye optical density for ethanol $/ \mathrm{D}_{2} \mathrm{O}$ (molar fraction of water $x_{w}=0.954$ ) are presented in Fig. 2, respectively. The contribution of the dye as a third component becomes weaker with decreasing dye content and disappears at low optical densities to the extend that a two mode analysis is no longer possible.

The increasing amplitude with dye content could be either due to screened electrostatic interactions by the charged dye molecules or due to convection problems, because the increasing dye optical density leads also to a larger temperature gradient. In order to investigate the role of convection we performed also laser intensity dependent measurements at constant dye content (c.f. Fig. 3). Extrapolation of both amplitude and diffusion coefficient to zero dye optical content (c.f. Fig. 2, $A_{\mathrm{OD} \rightarrow 0}=0.431, D_{\mathrm{OD} \rightarrow 0}=7.07 \times 10^{-6} \mathrm{~cm}^{2} \mathrm{~s}^{-1}$ ) and to zero laser intensity (c.f. Fig. $3, A_{\mathrm{OD} \rightarrow 0}=0.429, D_{\mathrm{OD} \rightarrow 0}=7.14 \times 10^{-6} \mathrm{~cm}^{2} \mathrm{~s}^{-1}$ ) agree within $0.5 \%$ and $1 \%$, respectively. Therefore it is sufficient for the aqueous systems to perform intensity dependent measurements at a low optical density around 2.5 and extrapolate to zero laser intensity. At the same time, the quantitative estimation of each contribution, convection 
and screening electrostatic interactions by dye molecules is difficult.

\section{RESULTS}

We performed systematic measurements of the Soret coefficient in different associated mixtures at different temperatures as a function of concentration. Fig. 4 shows the Soret coefficient for ethanol/water mixtures at different temperatures and concentrations. Figure 4a shows the influence of the temperature and Fig. 4b the effect of the isotopic substitution of $\mathrm{H}_{2} \mathrm{O}$ by $\mathrm{D}_{2} \mathrm{O}$. A positive Soret coefficient indicates that the ethanol molecules tend to move to the cold side. There are two characteristic points in Fig. 4a: the molar fraction of the solvent $x_{s}$ at which $S_{\mathrm{T}}$ changes its $\operatorname{sign} x_{s}^{ \pm}=0.142$ and the concentration at which $S_{\mathrm{T}}$ is not sensitive to the temperature $x_{s}^{\times}=0.142$ in the investigated temperature range between $T=25^{\circ} \mathrm{C}$ and $40^{\circ} \mathrm{C}$. In contrast to non-associated mixtures ${ }^{27}$ both concentrations are the same for this aqueous mixture. The isotopic shift of $\Delta S_{\mathrm{T}}=0.85 \times 10^{-3} \mathrm{~K}^{-1}$ (Fig. $4 \mathrm{~b}$ ) does not depend on the concentration which is in agreement with the results for non-associated mixtures ${ }^{26}$.

The data for DMSO/water are presented in Fig. 5 in the same way as for ethanol/water. Fig. 5a shows the temperature influence and Fig. 5b the effect of isotopic substitution. A positive Soret coefficient for DMSO in water implies that DMSO molecules accumulate at the cold side. The sign change concentration is also for this mixture not sensitive to the temperature $\left(x_{s}^{\times}=x_{s}^{ \pm}=0.195\right)$ and the isotopic shift of $\Delta S_{\mathrm{T}}=0.42 \times 10^{-3} \mathrm{~K}^{-1}$ is independent of concentration. Compared to the system ethanol/water the sign change concentration $x_{s}^{ \pm}=0.219$ for DMSO/water is higher. On the other hand the isotopic shift $\Delta S_{\mathrm{T}}$ is larger for the system ethanol/water compared to DMSO/water.

The Soret coefficient for 1-propanol/water at different temperatures and concentrations is plotted in Fig. 6. Also 1-propanol moves at low solvent content to the cold side, which is indicated by a positive Soret coefficient. In contrast to the systems ethanol/water and DMSO/water the sign change concentration depends on temperature and is equal to $x_{s}^{ \pm}$ $=0.078$ and 0.108 for 25 and $40^{\circ} \mathrm{C}$, respectively. For the investigated temperatures the intersection concentration of $x_{s}^{\times}=0.064$ is lower than $x_{s}^{ \pm}$.

Fig. 7 shows the Soret coefficient for methanol/DMSO and ethanol/DMSO at different concentrations and temperatures. The system methanol/DMSO does not show a sign 
change, while for the system ethanol/DMSO a sign change occurs at about $x_{\mathrm{DMSO}}^{ \pm}=0.4$ and 0.45 at 25 and $40^{\circ} \mathrm{C}$, respectively. For this system the sign change concentration varies with temperature. With increasing temperature the magnitude of $S_{\mathrm{T}}$ for methanol/DMSO mixture decreases for all concentrations by the same amount of $\Delta S_{\mathrm{T}} \simeq 0.5 \times 10^{-3} \mathrm{~K}^{-1}$. For ethanol/DMSO the temperature effect depends on the concentration and an intersection point can be observed in ethanol rich region, while the system methanol/DMSO does not have an intersection point at all.

\section{DISCUSSION}

Figures $4 \mathrm{a}$, 5a and 6 show the Soret coefficients for ethanol $/ \mathrm{H}_{2} \mathrm{O}, \mathrm{DMSO} / \mathrm{D}_{2} \mathrm{O}$ and 1propanol $/ \mathrm{H}_{2} \mathrm{O}$ mixtures at different concentrations and temperatures. For ethanol $/ \mathrm{H}_{2} \mathrm{O}$ and DMSO $/ \mathrm{D}_{2} \mathrm{O}$ mixtures $x^{\times}=x^{ \pm}$in the investigated temperature range, while for 1propanol $/ \mathrm{H}_{2} \mathrm{O} x^{\times} \neq x^{ \pm}$. In the following we discuss the characteristic points of the thermal diffusion behavior and relate them with the properties of the pure components and the mixture.

\section{A. The effect of temperature}

The observed independence of the sign change concentration on temperature for DMSO/water mixture is supported by a recent NMR study. Mizuno et al. ${ }^{34}$ measured the chemical shift of water hydrogen $\delta_{H_{2} O}$, which is related to polarization of water molecules, in dependence of concentration and temperature. In the investigated temperature range between $1-48.5^{\circ} \mathrm{C}$ the chemical shift for pure water is reached around $x_{w} \approx 0.8$. Then the chemical shift slightly overshoots the water value indicating that DMSO stabilizes the water structure. Therefore the stabilized hydrogen bond network forms temperature independent between $x_{w} \approx 0.8$ and 1.0, suggesting $x^{\times}=x^{ \pm}$in the investigated temperature range. Also for the system acetone/water a stronger polarization of the water molecules in the vicinity of acetone has been observed at high water content $x_{w}>0.96^{35}$. So the polarization effect occurs at higher water content compared to DMSO/water, but it shows in the investigated temperature range no temperature dependence. The thermal diffusion behavior of those two systems follows a similar trend with respect to temperature, whereas the water content at 
the sign change concentration is higher for acetone/water than for DMSO/water.

Takamuku et al. ${ }^{36}$ investigated aqueous solutions at low temperatures by X-ray scattering. They found, that for methanol/water mixture the composition of the dominant clusters formed at $25^{\circ} \mathrm{C}$ does not significantly change when the temperature is lowered. In contrast, 2propanol/water shows a stronger temperature dependence. For 2-propanol the chain clusters are enhanced in the mixture when the temperature is lowered. This might be an indication why for systems with stronger hydrophobic interactions the sign change concentration of the Soret coefficient can be temperature dependent.

\section{B. Relation between the thermal diffusion motion and a structural change in the}

\section{fluid}

At high water content for all aqueous systems studied so far water accumulates at the warm side. In many cases the Soret coefficient changes its sign at a certain solvent concentration $x_{s}^{ \pm}$. For the systems regarded here we observe the following sequence $x_{s}^{ \pm}=0.08,0.095,0.11,0.14,>0.18$ and 0.195 for 1-propanol, 2-propanol, acetone, ethanol, methanol and DMSO in water. In the case of methanol/water the sign change concentration could not be determined precisely from the literature data ${ }^{37}$ and also TDFRS measurements were not possible in the entire concentration range due to the low refractive index contrast between methanol and water. Therefore, we estimated the highest concentration of methanol, below which no sign change occurs by measuring diluted aqueous solutions.

It is intuitive to relate the sign change concentration with structural changes in the fluid mixture. There are numerous nuclear magnetic resonance (NMR) ${ }^{34}$, mass spectrometry ${ }^{38}$, dielectric spectroscopy ${ }^{39}$ and x-ray scattering ${ }^{40}$ studies, which investigate the change from the tetrahedral structure of water to other structures such as chain-like conformations with increasing solvent concentration. Takamuku et al. ${ }^{40}$ investigated alcohol/water solutions by $\mathrm{X}$-ray scattering and found that the structure changes with increasing alcohol concentration in the order of n-propyl $\approx$ iso-propyl $>$ ethyl $>$ methyl at alcohol mole fractions of $0.1,0.1$, 0.2 , and 0.3 , respectively. Additionally, the same concentrations correspond to the minima of the enthalpies of mixing of the alcohol/water mixtures, which is explained by a compensation of an enthalpic gain due to alcohol/water hydrogen bonding with an enthalpic loss due to a breaking of the tetrahedral water structure with increasing alcohol concentration. 
Thus, the larger the hydrophobic group, the more rapidly the tetrahedral-like structure of water is disturbed with increasing alcohol concentration. We observed the same tendency for the sign change concentrations for aqueous mixtures of 1-propanol, 2-propanol, ethanol and methanol. The small difference in the thermal diffusion behavior between 1-propanol and 2-propanol might be explained by the observation, that the minimum of the excess enthalpy for 1-propanol/water mixture is shifted more towards the water rich region compared to 2-propanol/water mixture ${ }^{39}$, which suggests that the water structure is more effectively disrupted by 1-propanol. The structural changes in DMSO/water mixtures have been investigated by Shin et al. ${ }^{38}$ by mass spectrometry. They observed that the water clusters disappear between $x_{s}=0.19$ and 0.25 , which is the concentration range, in which the Soret coefficient changes it's sign. The structural change is also supported by the NMR-study by Mizuno et al. ${ }^{34}$ which has been discussed in Sec. IV A. They found that the chemical shift of methyl hydrogen decreased below a $x_{w}=0.8$ indicating a disruption of the tetrahedral water structure. This supports our hypothesis that the direction of the thermal diffusion is correlated with the fluid structure - indicated by the formation of water clusters - on a microscopic level.

\section{The effect of solubility}

The absolute value of the Soret coefficient was previously connected with the solubility parameter $^{21}$. Mutually soluble components become undistinguishable, so that the Soret coefficient of such mixtures is equal to zero. Consequently, the Soret coefficient should increase with decreasing solubility. Generally, the solubility is determined by the Gibbs mixing energy $\left(\Delta G_{M}=\Delta H_{M}-T \Delta S_{M}\right)$. With increasing temperature enthalpy $\left(\Delta H_{M}\right)$ and entropy $\left(-T \Delta S_{M}\right)$ contributions to the Gibbs energy act in the opposite way (as far as both $\Delta H_{M}$ and $\Delta S_{M}$ become larger due to weaker hydrogen bonding and less ordering in the system, respectively). For ethanol/water and DMSO/water mixtures the increasing temperature leads probably to an increasing solubility ${ }^{41}$, which decreases the absolute values of the Soret coefficient in the whole concentration range (c.f. Fig. 4a, 5a). This is not the case for water propanol mixtures (c.f. Fig. 6). The additional contribution in enthalpy/entropy competition due to the enhancement of propanol clusters ${ }^{40}$ could be responsible for the observed behavior (c.f. Fig. 6). 


\section{The effect of hydrophilic interactions}

As we already mentioned the sign change concentration can be attributed to the stability of water clusters. At the same time, the stability of water clusters is determined by the hydrophilic (dipole moment) and hydrophobic (number of carbon atoms in hydrophobic part) parts of the solute molecules. In order to study the effect of hydrophilic interactions we consider aqueous mixtures of solvents with the same hydrophobic part (two carbon atoms). In the case of acetone and propionaldehhyde the third carbon is polarized by the double bond with the oxygen and not counted. With the assumption that the intermolecular interactions can be characterized by the enthalpy of vaporization, the stability of water clusters can be determined by the ratio of vaporization enthalpy of the solvent and water. The ratio of the vaporization enthalpies can also be attributed to the ratio of pure interaction parameters of a Lennard-Jones (LJ) mixture (e.g. $\left.\psi=H_{2}^{v a p} / H_{1}^{v a p}=\varepsilon_{22} / \varepsilon_{11}\right)^{21}$. Artola et al. ${ }^{42}$ investigated the concentration dependence of the Soret coefficient for binary LJ mixtures of particles with the same masses and sizes but different strength of direct $\psi=\varepsilon_{22} / \varepsilon_{11}$ and cross interaction

energies $k_{12}=\varepsilon_{12} \sqrt{\left(\varepsilon_{22} / \varepsilon_{11}\right)}$, respectively. The slope of the composition dependence $S_{\mathrm{T}}(x)$ is controlled by the value of $k_{12}$. This corresponds with a variation of the temperature (c.f. Fig. $4 \mathrm{a}$ and 5a). In contrast $\psi$, which is equivalent to the ratio of vaporization enthalpies, has almost no effect on the slope, but it shifts the Soret coefficient as mass and inertia changes do. The latter was already noticed by Köhler et al. ${ }^{43}$. In Fig. 8 the sign change concentration is plotted versus the ratio of the vaporization enthalpies $H_{s}^{\text {vapor }} / H_{w}^{\text {vapor }}$ of the pure components, which is equivalent to the $\psi$ parameter. Data for aqueous solutions of solutes with a similar hydrophobic part (propionaldehyde, acetone, DMSO, ethanol) follow the straight line, which indicates for those systems the importance of hydrophilic interaction rather than effect of mass or moment of inertia. The isotopic substitution of water decrease $x_{s}^{ \pm}$, but the roughly $10 \%$ larger vaporization energy for heavy water compensates this effect so that also those systems follow the line. At the same time decreasing (or increasing) the hydrophobic part of the solute increases in case of methanol (or decrease in case of propanol) the concentration at which the sign change occurs. A similar trend has been observed for the break down of the hydrogen bond network ${ }^{40}$. Additionally, our measurements suggest that for all systems on the line the sign change concentration is temperature independent, $x_{s}^{ \pm}=x^{\times}$. 


\section{E. Effects in alcoholic mixtures with DMSO}

The system methanol/DMSO stands out from the other systems because it has neither an intersection point nor a point when the Soret coefficient changes its sign. In a recent molecular dynamic simulation by Vechi and Skaf ${ }^{44}$ it was shown that the structure formation in methanol/DMSO mixtures is not markedly altered by changing the concentration. This is an indication that DMSO and methanol are quite similar and compatible, which might also be the reason that the sign of the Soret coefficient does not change in dependence of concentration.

The stability of DMSO clusters influences the thermal diffusion behavior of both mixtures. The absence of the sign change for methanol/DMSO mixtures means better miscibility of methanol (no DMSO cluster) in comparison with ethanol (DMSO clusters form) in DMSO. Unfortunately, we did not find a value for the Gibbs energy of mixing, but the enthalpic contribution is negative for methanol/DMSO and positive for ethanol/DMSO mixtures ${ }^{45}$, which indicates a better solubility of methanol in DMSO.

The difference between the systems methanol/DMSO and ethanol/DMSO mixtures lies in their different hydrophilic groups. As stated already methanol is very similar to DMSO and the fluid structure does not depend on the mixing ratio. The similarity of the two substances is also reflected by the similar hydrogen bonding parameters of DMSO and methanol, which are almost twice as large as the one for ethanol ${ }^{46}$. Therefore it is likely that ethanol will introduce a different fluid structure due to the larger hydrophobic group which leads then to a sign change of the Soret coefficient.

\section{CONCLUSION}

We studied systematically the thermal diffusion behavior of associated and highly polar mixtures. Many of those mixtures show a sign change of $S_{\mathrm{T}}$ with concentration. In the case of aqueous mixtures the sign changes occurs at high water content between $x_{w}^{ \pm}=$ $0.8-0.92$. For many systems this concentration can be related with a structural change from a tetrahedral to a chain-like order. Another characteristic point of the investigated systems is the concentration $x_{w}^{\times}$at which the Soret coefficient does not depend on temperature in the investigated range. While for non-associating mixtures $S_{\mathrm{T}}\left(x_{w}^{\times}\right) \neq 0$ the Soret coefficient 
vanishes often for associating mixtures $\left(S_{\mathrm{T}}\left(x_{w}^{\times}\right) \neq 0\right)$. For those systems with $x_{w}^{ \pm}=x_{w}^{\times}$ we found that $x_{w}^{ \pm}$depends linearly on the ratio of the vaporization enthalpies of the pure components. Additionally the hydrophobic part of those systems consists of two carbon atoms.

We also investigated two polar but non-aqueous mixtures: methanol/DMSO and ethanol/DMSO. The system methanol/DMSO does neither show a sign change nor an intersection point of the isotherms, which might be due to the similarity of methanol and DMSO. Ethanol/DMSO behaves like a typical non-associating mixture with $x_{w}^{ \pm} \neq x_{w}^{\times}$. To reveal further the microscopic mechanism of the thermal diffusion process molecular dynamic studies need to be performed.

\section{ACKNOWLEDGMENTS}

We would like to thank the Deutsche Forschungsgemeinschaft for the financial support (WI 1684) and we appreciate fruitful discussions with Malte Kleemeier, Florian MüllerPlathe, and Bernard Rousseau.

\footnotetext{
* p.polyakov@fz-juelich.de; http://www.fz-juelich.de/iff/personen/P.Polyakov/

$\dagger$ s.wiegand@fz-juelich.de; http://www.fz-juelich.de/iff/personen/S.Wiegand/

1 M. E. Schimpf and J. C. Giddings, Macromolecules 20, 1561 (1987).

2 K. Clusius and G. Dickel, Naturwissenschaften 27, 148 (1939).
}

3 H. C. Helgeson, Pure \& Appl. Chem. 57, 31 (1885).

4 P. Costeseque, D. Fargue, and P. Jamet, in Thermal nonequilibrium phenomena in fluid mixtures, edited by W. Köhler and S. Wiegand (Springer, Berlin, 2000), Lecture Notes in Physics, pp. 389-427.

5 D. E. Rosner, R. S. Israel, and B. La Mantia, Combustion and Flame 123, 547 (2000).

6 C. Ludwig, Sitz. ber. Akad. Wiss. Wien Math.-naturw. Kl 20, 539 (1856).

7 O. Ecenarro, J. A. Madariaga, J. Navarro, C. M. Santamaria, J. A. Carrion, and J. M. Saviron, J.Phys.:Condens. Matter 2, 2289 (1990).

8 W. Köhler and B. Müller, J. Chem. Phys. 103, 4367 (1995). 
9 W. B. Li, P. N. Segre, R. W. Gammon, J. V. Sengers, and M. Lamvik, J. Chem. Phys. 101, 5058 (1994).

10 K. J. Zhang, M. E. Briggs, R. W. Gammon, and J. V. Sengers, J. Chem. Phys. 104, 6881 (1996).

11 M. M. Bou-Ali, O. Ecenarro, J. A. Madariaga, C. M. Santamaria, and J. J. Valencia, J.Phys.:Condens. Matter 10, 3321 (1998).

12 J. K. Platten, M. M. Bou-Ali, and J. F. Dutrieux, Philos. Mag. 83, 2001 (2003).

13 P. Kolodner, H. Williams, and C. Moe, J. Chem. Phys. 88, 6512 (1988).

14 J. F. Dutrieux, J. K. Platten, G. Chavepeyer, and M. M. Bou-Ali, J. Phys. Chem. B 106, 6104 (2002).

15 R. Kita, S. Wiegand, and J. Luettmer Strathmann, J. Chem. Phys. 121, 3874 (2004).

16 H. Ning, R. Kita, and S. Wiegand, Progr. Colloid Polym. Sci. 133, 111 (2006).

17 H. Ning, R. Kita, H. Kriegs, J. Luettmer-Strathmann, and S. Wiegand, J. Phys. Chem. B 110, $10746(2006)$.

18 S. Wiegand, J.Phys.:Condens. Matter 16, R357 (2004).

19 D. Reith and F. Müller-Plathe, J. Chem. Phys. 112, 2436 (2000).

20 G. Galliero, B. Duguay, J. P. Caltagirone, and F. Montel, Fluid Phase Equilibr. 208, 171 (2003).

21 P. Polyakov, M. Zhang, F. Müller-Plathe, and S. Wiegand, J. Chem. Phys. 127, 014502 (2007).

22 V. Dorogush, Inzh.-Phys. Zh. 41, 503 (1981).

23 D. Stanford, J. Chem. Phys. 58, 4338 (1973).

24 G. Wittko and W. Köhler, J. Chem. Phys. 123, 014506 (2005).

25 P. Polyakov, J. Luettmer-Strathmann, and S. Wiegand, J. Phys. Chem. B 110, 26215 (2006).

26 G. Wittko and W. Köhler, J. Chem. Phys. 123, 014506 (2005).

27 G. Wittko and W. Köhler, Europhys. Lett. 78, 46007 (2007).

28 H. Ning and S. Wiegand, J. Chem. Phys. 125, 221102 (2006).

29 J. Johnson and A. Beyerlein, J. Phys. Chem 82, 1430 (1978).

30 N.-Y. Ma, D. Stanford, and A. Beyerlein, J. Phys. Chem 87, 5464 (1983).

31 S. Wiegand, R. Kita, H. Ning, and S. Wiegand, J. Noneq. Thermod 32, 193 (2007).

32 A. Becker, W. Köhler, and B. Müller, Ber. Bunsen-Ges-Phys. Chem. 99, 600 (1995).

33 A. Perronace, C. Leppla, F. Leroy, B. Rousseau, and S. Wiegand, J. Chem. Phys. 116, 3718 (2002). 
34 K. Mizuno, S. Imafuji, T. Ochi, T. Ohta, and S. Maeda, J. Phys. Chem. B 104, 11001 (2000).

35 K. Mizuno, T. Ochi, and Y. Shindo, J. Chem. Phys. 109, 9502 (1998).

36 T. Takamuku, K. Saisho, S. Nozawa, and T. Yamaguchi, J. Molec. Liq. 119, 133 (2005).

37 L. J. Tichacek, W. S. Kmak, and H. G. Drickamer, J. Phys. Chem. 60, 660 (1956).

38 D. N. Shin, J. W. Wijnen, J. Engberts, and A. Wakisaka, J. Chem. Phys. B 105, 6759 (2001).

39 T. Sato and R. Buchner, J. Chem. Phys. 119, 10789 (2003).

40 T. Takamuku, H. Maruyama, K. Watanabe, and T. Yamaguchi, J. Solution Chem. 33, 641 (2003).

41 V. P. Belousov and M. Y. Panov, Thermodynamic properties of aqueous solutions of organic substances. (CRC Press, Boca Raton, FL, 1994).

42 P.-A. Artola and B. Rousseau, Phys. Rev. Lett. 98, 125901 (2007).

43 C. Debuschewitz and W. Köhler, Phys. Rev. Lett, 87, art. no. (2001).

44 S. M. Vechi and M. S. Skaf, J. Chem. Phys. 123 (2005).

45 K. Rubini, R. Francesconi, A. Bigi, and F. Comelly, Thermochmica Acta 452, 124 (2007).

46 A. F. M. Barton, ed., CRC Handbook of Solubility Parameters and Other Cohesion Parameters, vol. 2nd ed (CRC Press Inc., Boca Laton, FL, 1991). 


\section{List of Figures}

Figure 1: (a) Typical normalized TDFRS signals for an ethanol/ $\mathrm{D}_{2} \mathrm{O}$ mixture $\left(x_{w}=0.954\right)$ at different dye optical densities $\mathrm{OD}=9.8 \mathrm{~cm}^{-1}(\boldsymbol{\square})$ and $\mathrm{OD}=2.4 \mathrm{~cm}^{-1}(\mathbf{O})$, respectively. (b) The residual plot according to the one mode (Eq. 2, $\mathbf{\square})$ and the two mode analysis ${ }^{17}$ (Eq. $3, \square)$ for ethanol $/ \mathrm{D}_{2} \mathrm{O}$ at a high dye content $\left(\mathrm{OD}=9.8 \mathrm{~cm}^{-1}\right)$.

Figure 2: Careful check of the influence of the dye on the signal. Amplitude and diffusion coefficient $D$ of ethanol $/ \mathrm{D}_{2} \mathrm{O}$ mixtures $\left(x_{w}=0.954\right)$ as a function of the dye optical density. Solid $(\mathbf{\square}, \mathbf{O})$ and open $(\square)$ data points are obtained from the one (c.f. Eq. 2) and two mode (c.f. Eq. 3) analysis, respectively. With a linear fit (solid line) the corresponding amplitude and diffusion coefficient can be determined at $\mathrm{OD}=0$.

Figure 3: Amplitude (ם) and diffusion coefficient $D(\mathbf{O})$ of ethanol $/ \mathrm{D}_{2} \mathrm{O}$ mixtures at a molar fraction of water $x_{w}=0.954$ as function of the laser intensity in front of the cell at $\mathrm{OD}=3.7 \mathrm{~cm}^{-1}$.

Figure 4: (a) Soret coefficient $S_{\mathrm{T}}$ of ethanol $/ \mathrm{H}_{2} \mathrm{O}$ mixtures at three different temperatures $22.5^{\circ} \mathrm{C}(\boldsymbol{\square}), 32.5^{\circ} \mathrm{C}(\boldsymbol{O})$ and $42.5^{\circ} \mathrm{C}(\boldsymbol{\Delta})$. The open symbols are data from Kolodner et. al ${ }^{13}$ at $20(\square)$ and $40^{\circ} \mathrm{C}(\triangle)$. (b) Soret coefficient $S_{\mathrm{T}}$ of ethanol $/ \mathrm{H}_{2} \mathrm{O}(\diamond)$ and ethanol $/ \mathrm{D}_{2} \mathrm{O}(\boldsymbol{\square})$ mixtures at $22.5^{\circ} \mathrm{C}$.

Figure 5: (a) Soret coefficient $S_{\mathrm{T}}$ of $\mathrm{DMSO} / \mathrm{D}_{2} \mathrm{O}$ mixtures at $25^{\circ} \mathrm{C}(\boldsymbol{\square})$ and $45^{\circ} \mathrm{C}(\boldsymbol{\Delta})$. (b) Soret coefficient $S_{\mathrm{T}}$ of DMSO $/ \mathrm{H}_{2} \mathrm{O}^{28}(\square)$ and DMSO $/ \mathrm{D}_{2} \mathrm{O}(\boldsymbol{O})$ mixtures at the temperature of $25^{\circ} \mathrm{C}$. The error bars do not exceed the symbol size

Figure 6: Soret coefficient $S_{\mathrm{T}}$ of 1-propanol/water mixture at $25^{\circ} \mathrm{C}(\boldsymbol{\square})$ and $40^{\circ} \mathrm{C}(\boldsymbol{\Delta})$. Figure 7: Soret coefficient $S_{\mathrm{T}}$ of ethanol/DMSO $(\square, \bigcirc)$ and methanol/DMSO $(\mathbf{\square}, \mathbf{0})$ mixtures at different concentrations at $25^{\circ} \mathrm{C}(\square, \mathbf{\square})$ and $40^{\circ} \mathrm{C}(\bigcirc, \mathbf{O})$.

Figure 8: Sign change concentration $x_{s}^{ \pm}$plotted versus the ratio of the vaporization enthalpies of the pure components. For aqueous systems where the solvent has two carbons $(\diamond, \diamond)$ $x_{s}^{ \pm}$increases linearly with the ratio $H_{s}^{\text {vapor }} / H_{w}^{\text {vapor }}$, while solvents with three carbon atoms (৩) do not follow the line. Some data for DMSO $/ \mathrm{H}_{2} \mathrm{O}(\diamond)$ and acetone $/ \mathrm{H}_{2} \mathrm{O}(\diamond)$ have been taken from the literature ${ }^{28}$. 
Figures

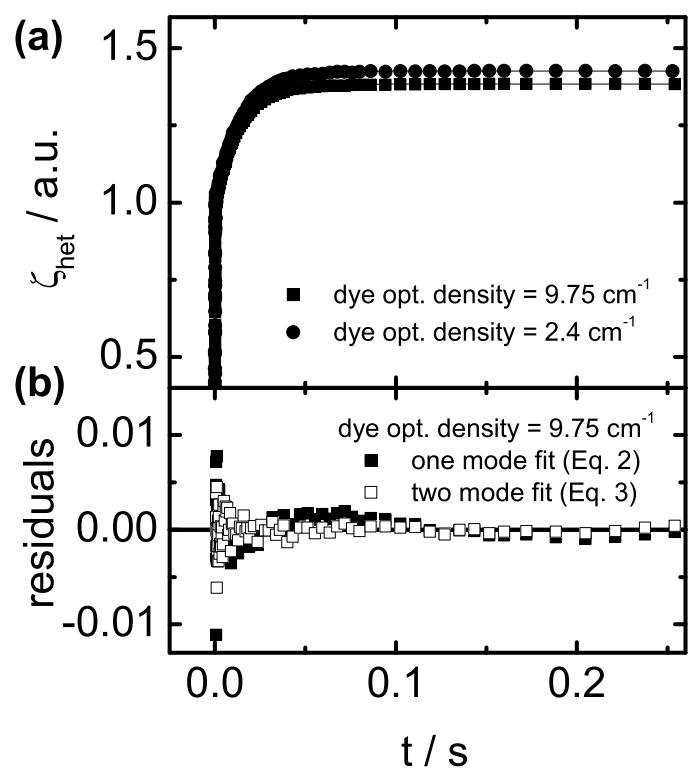

FIG. 1: 


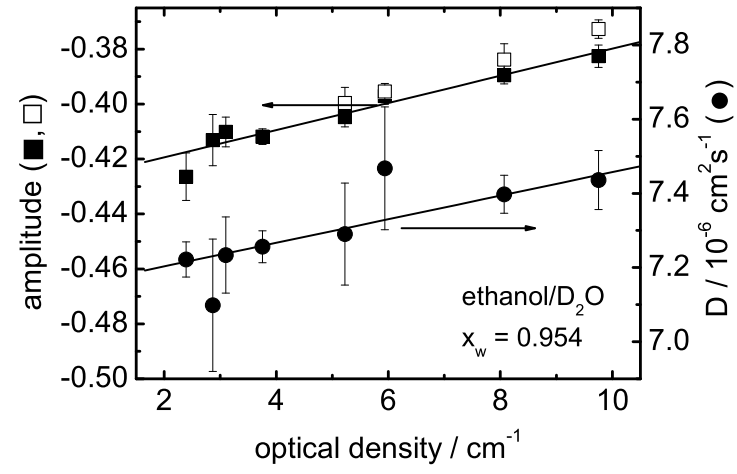

FIG. 2: 


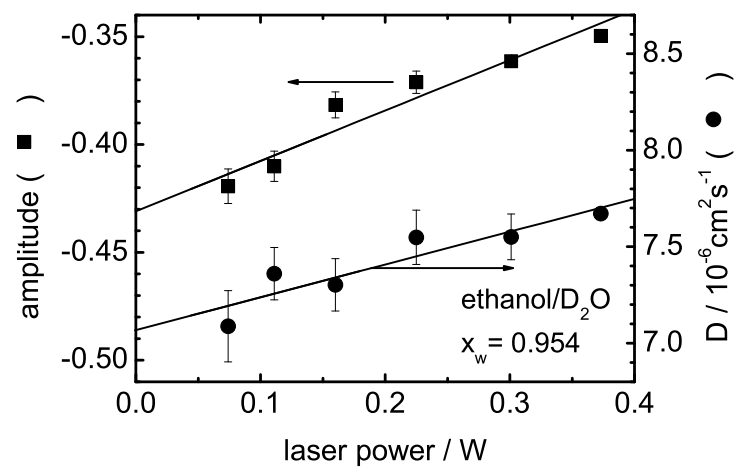

FIG. 3: 


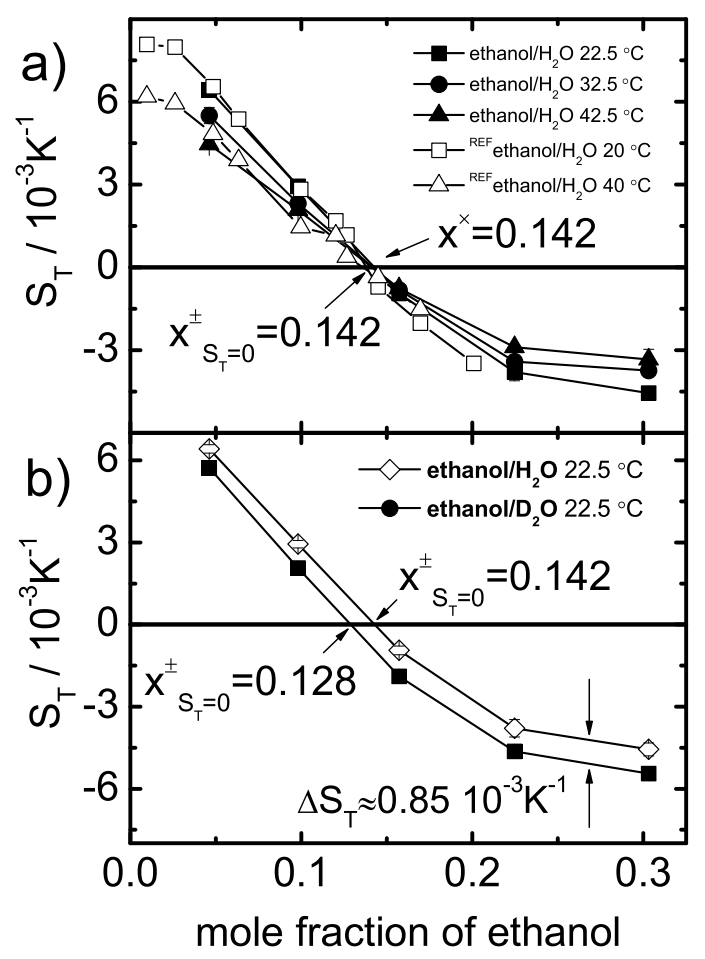

FIG. 4: 


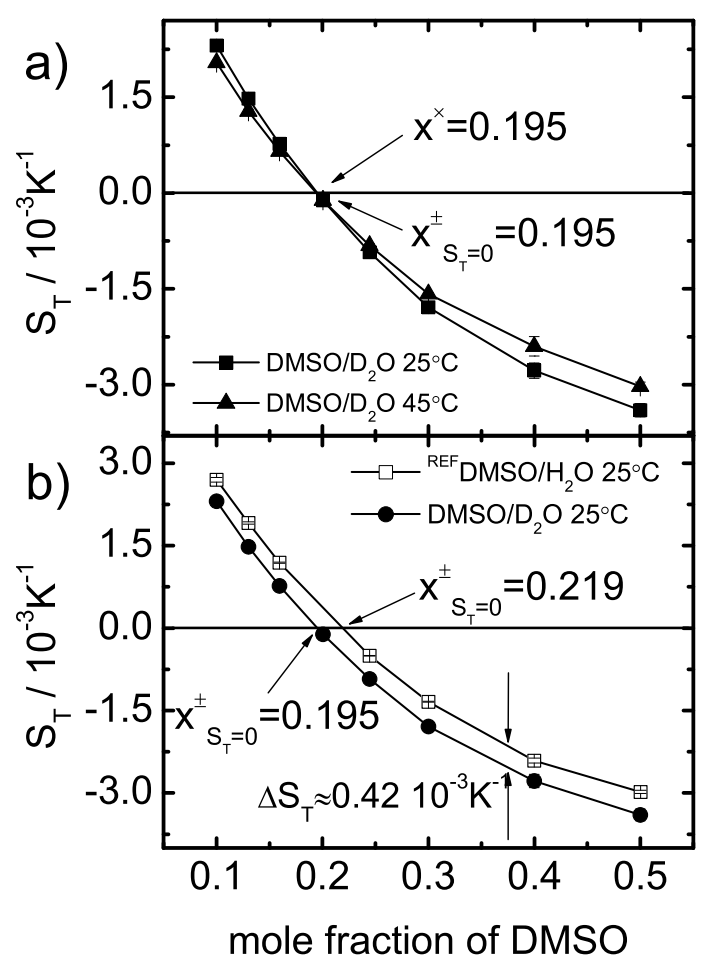

FIG. 5: 


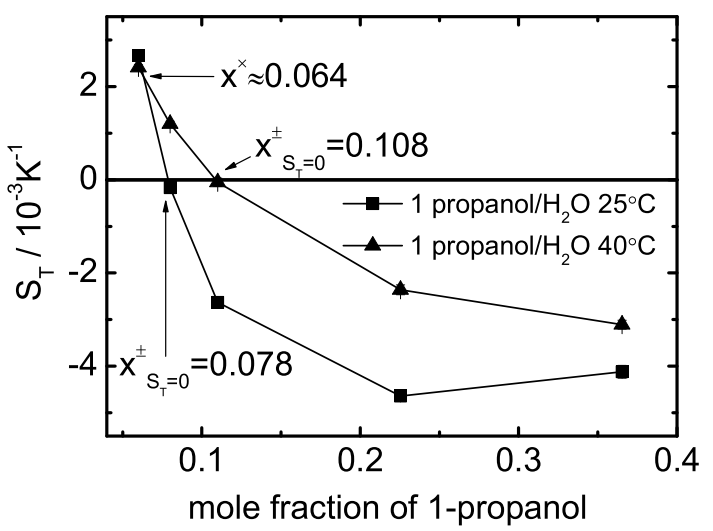

FIG. 6: 


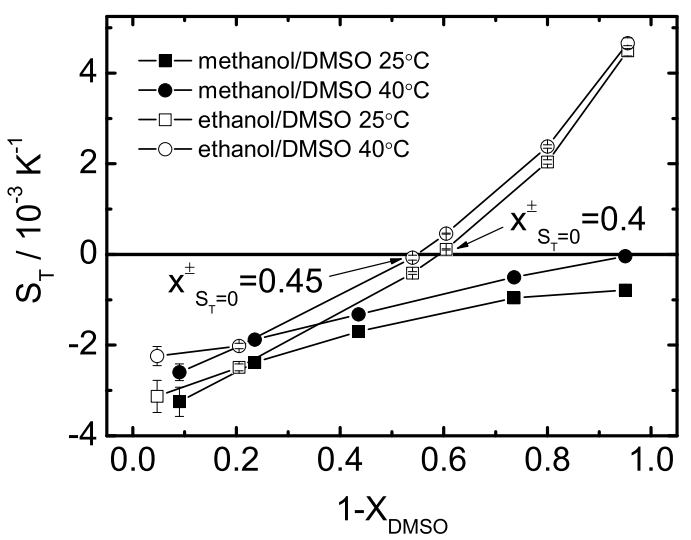

FIG. 7: 


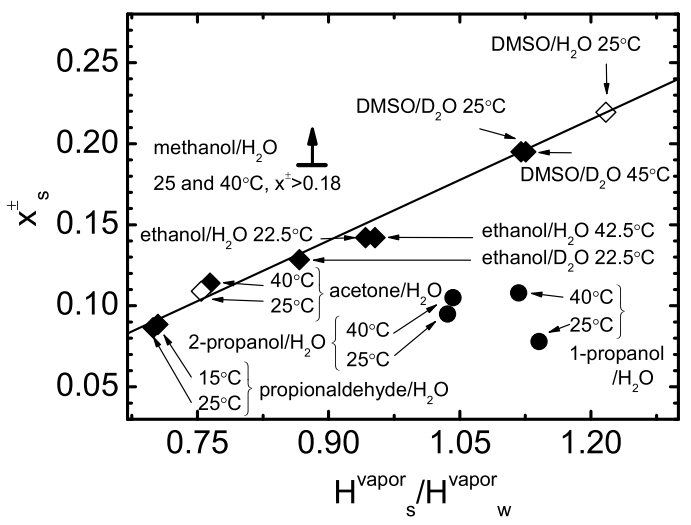

FIG. 8: 